\title{
Synthesis of Pyrazoles by Three-Component One-Pot Process
}

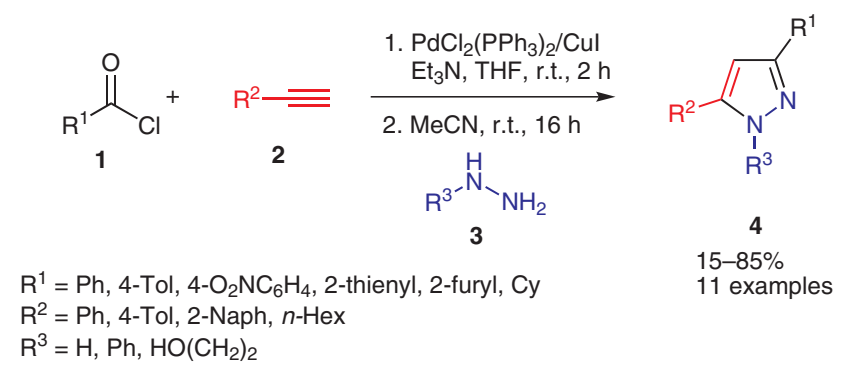

Significance: The one-pot synthesis of pyrazoles by Sonogashira-type reaction of acyl chlorides $\mathbf{1}$ with substituted acetylenes $\mathbf{2}$, followed by cyclocondensation in presence of various hydrazines $\mathbf{3}$ is reported. The procedure is rather simple and convenient; however, it requires optimization, and preparative yields vary from poor to excellent. The method may be used for pyrimidine synthesis as well, as shown for a single example. In this case acetamidine hydrochloride $\mathbf{7}$ serves as a third coupling reagent.
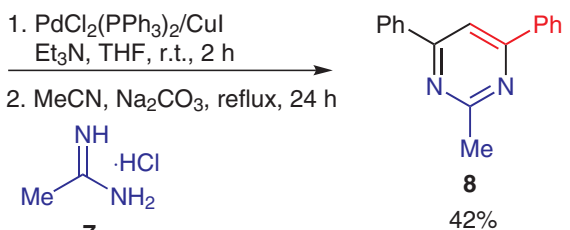

Comment: Nitrogen-containing heterocycles in general and pyrazole and pyrimidine derivatives specifically possess important biological and pharmaceutical activities and are also useful synthetic building blocks in organic chemistry. The proposed method uses easily available cheap starting materials, combines simplicity of procedures with reasonable preparative yields. For very recent studies of pyrazole-based bioactive materials, see: P. Singh, K. Paul, W. Holzer Bioorg. Med. Chem. 2006, 14, 5061; C. Lamberth Heterocycles 2007, 71, 1467. 